\title{
New Method to Generate Reversible Hydrophobic and Hydrophilic Surfaces
}

\author{
V. Mittal ${ }^{*}, 1, \#$ and N.B. Matsko ${ }^{2}$ \\ ${ }^{I}$ Department of Chemistry and Applied Biosciences, Institute of Chemical and Bioengineering, ETH Zurich, 8093 \\ Zurich, Switzerland \\ ${ }^{2}$ Electron Microscopy Center ETH Zurich (EMEZ), ETH Zurich, 8093 Zurich, Switzerland
}

\begin{abstract}
Designer particles synthesized by surfactant-free emulsion polymerization were adsorbed successfully on the sapphire discs. The particles carried a thin layer of an atom transfer radical polymerization (ATRP) initiator on the surface and were subsequently successfully used to graft PNIPAAM brushes from the surface. The surface coverage of the discs by these particles could be improved by heat treatment of the discs which fused the particles with each other but keeping the initial ATRP layer on them. The reversible wetting characteristics of the surfaces as a function of temperature could be established by studying the behavior of water droplets placed on the PNIPAAM modified discs. The heat treated discs improved the reversible behavior further, though no superhydrophobic and superhydrophilic behavior could be achieved. The reversible temperature responsively makes these surfaces tremendously important for the commercial separation and filtration processes.
\end{abstract}

Keywords: Atom transfer radical polymerization, poly (N-isopropylacrylamide), sapphire discs, polystyrene, hydrophobicity, hydrophilicity.

\section{INTRODUCTION}

Generation of surfaces with specific functionalities has always been a focus of interest. One such category of tremendous interest is represented by reversible hydrophilic and hydrophobic surfaces. To this regard, poly $(\mathrm{N}-$ isopropylacrylamide) (PNIPAAM) has received particular attention, being a water soluble polymer with a lower critical solution temperature (LCST) of about $32^{\circ} \mathrm{C}$ [1-6]. Below the LCST, the polymer chains exhibit chain extended conformations and random coil structure. The intermolecular hydrogen bonding with the water molecules due to the chain extended morphology generates the hydrophilic nature of the chains. Above LCST, the chains transform into more collapsed globular form. The intramolecular bonding between the $\mathrm{CO}$ and $\mathrm{NH}$ groups dominate over the external hydrogen bonding (Fig. 1). Recently, superhydrophobic and superhydrophilic surfaces were reported when the PNIPAAM chains were grown on extremely rough surfaces [7]. Contact angles with water of $\approx 0^{\circ}$ and $\approx 150^{\circ}$ were reported at $25^{\circ} \mathrm{C}$ and $40^{\circ} \mathrm{C}$ respectively. However, one drawback of such techniques to achieve functional surfaces is the high cost involved in the surface preparation techniques owing to the use of laser ablation; photolithography based modified surfaces etc $[8,9]$. This hinders the use of such useful surfaces for the large scale production. Therefore, synthetic routes which can provide the cost reduction are necessary to be developed in order to achieve commercial application of these reversible hydrophobic and hydrophilic surfaces.

\footnotetext{
*Address correspondence to this author at the Department of Chemistry and Applied Biosciences, Institute of Chemical and Bioengineering, ETH $\mathrm{Zu}-$ rich, 8093 Zurich, Switzerland; Tel: +41-44-632 5688; Fax: +41-44-632 1082; E-mail: vikas.mittal@chem.ethz.ch

${ }^{\#}$ Current Address: BASF SE, Germany
}

Recently, some studies focused on the grafting of PNIPAAM brushes from the surfaces of the spherical latex particles in order to achieve well defined brush architectures by using atom transfer radical polymerization of NIPAAM [1015]. To achieve this, particle surfaces have been functionalized with a thin layer of ATRP initiator beforehand. Thermally reversible brushes could successfully be synthesized. As these particles are inexpensive as compared to the costly laser treatment techniques, it would be an added advantage if these particles can be made to generate the thermally responsive surfaces. This would not only simplify the process, but also would cut down the costs tremendously. Much better control of the surface properties can also be achieved by controlling the properties of the latex particles beforehand. As the hydrophobic and hydrophilic behavior of the surfaces was reported to be enhanced by the roughness of the surface, the polymer particles owing to their rough surface morphology can also be expected to induce similar behavior.

This paper aims to present a different approach to develop thermally responsive surfaces. It includes adsorption of functional latex particles on the surfaces and subsequent polymerization of NIPAAM from the surface to generate thermally responsive behavior. Sapphire discs were used as substrates. Response of the PNIPAAM modified surfaces to temperatures was analyzed by the behavior of water droplets placed on them which was captured by a CCD camera.

\section{EXPERIMENTAL}

\section{a) Materials}

Styrene $(\mathrm{S}, \geq 99.5 \%)$ and potassium peroxodisulphate (KPS, $>99.0 \%$ ) were purchased from Fluka (Buchs, Switzerland) and were used as supplied without further purifications. ATRP initiator end capped with an acrylic moiety (2(2-bromopropionyloxy) ethyl acrylate, BPOEA) was synthe- 
sized as reported earlier [16]. N-isopropylacrylamide (NIPAAM, 97\%) and other reagents to run the ATRP polymerization, namely 1,1,4,7,10,10-hexamethyltriethylenetetramine (HMTETA, 97\%), copper(I) bromide (CuBr, 99.99\%), copper(II) bromide $\left(\mathrm{CuBr}_{2}, 99.99 \%\right) \&$ powder copper $(\mathrm{Cu}$, 99\%, 200 mesh) were procured from Aldrich (Buchs, Switzerland). Ultra pure Millipore water was employed in all experiments.

\section{b) Synthesis of Polystyrene Particles and their Surface Functionalization}

Polystyrene seed latex was prepared by surfactant free emulsion polymerization of styrene $(14 \mathrm{~g})$ added with $0.3 \mathrm{~g}$ KPS and $310 \mathrm{~g}$ of water.14 Surface functionalization of seed particles were carried out as reported earlier by generating a thin shell of acrylic end capped ATRP initiator (BPOEA) around them [14]. For this, $15 \mathrm{~g}$ of latex was heated to $70^{\circ} \mathrm{C}$ at $400 \mathrm{rpm}$ and purged with alternate vacuum/nitrogen cycles. BPOEA $(0.21 \mathrm{~g})$ was added to the latex followed by KPS solution ( $0.0025 \mathrm{~g}$ of KPS in $0.5 \mathrm{ml}$ of water) after 15 minutes. The reaction was allowed to run for $5 \mathrm{~h}$. Finally, the functionalized latexes were washed by repeated ultracentrifugation and resuspension in millipore water cycles.

\section{c) Adsorption of Latex Particles on the Sapphire Disks}

Carbon coated sapphire discs, freshly etched by charged oxygen plasma (10 sec, $100 \mathrm{mV}, 5$ mbar of $\mathrm{O}_{2}$ ) in Balzers GEA-003-S glow-discharge apparatus (Balzers), were placed on the droplet of particle latex for $2 \mathrm{~min}$ and dried on filter paper. In another approach to adsorb the particles, sapphire discs were treated with positive plasma to generate positive charges on the surface. The discs were similarly placed on the droplet of particle latex for $2 \mathrm{~min}$ and dried on filter paper. Adsorption studies were carried out as a function of temperature also. For this, the discs after particle adsorption were placed on the heating plates equilibrated at set temperatures for 60 seconds. Grids were prepared at room temperature, $100^{\circ} \mathrm{C}$ and $150^{\circ} \mathrm{C}$.

\section{d) Electron Microscopy}

The surface morphology of the adsorbed particles on the sapphire disks was observed in Hitachi field emission in-lens S-900 high resolution scanning electron microscope at accelerating voltages of $10-20 \mathrm{kV}$. The discs after adsorption and thermal treatment were sputter coating with $3 \mathrm{~nm}$ thick platinum layers and analyzed.

\section{e) Atom Transfer Radical Polymerization on the Sap- phire Disks}

Growth of PNIPAAM chains from the discs adsorbed with functionalized latex particles was achieved by placing the monoliths in an aqueous solution of NIPAAM, HMTETA, $\mathrm{CuBr}, \mathrm{CuBr}_{2}$ and $\mathrm{Cu}$ powder followed by degassing and purging with nitrogen. The disks were kept immersed in the monomer solution overnight and were subsequently placed in Millipore water 5-7 times to wash off any unreacted monomer.

\section{f) Study of the Behavior of Water Droplets on PNIPAAM Modified Sapphire Discs with Temperature}

The behavior of the water droplets on the sapphire discs functionalized with PNIPAAM chains at room temperature and at $40^{\circ} \mathrm{C}$. Images of the droplets were recorded using a Sony CCD camera (DFW-V 500, Japan) at a rate of 40 frames per second. The disks were placed on the surfaces set at required temperature for $5 \mathrm{~min}$ to equilibrate the disk surface and the water droplet $(\approx 5 \mu \mathrm{L})$ was placed subsequently on it. The droplets behavior was through the camera and the stills at time $\mathrm{t}=5 \mathrm{sec}$ are reported as a result. Each droplet was observed on the disk for two minute in order to analyze the effect on time on the droplet morphology.

\section{RESULTS AND DISCUSSION}

Thermally responsive surfaces have tremendous application potential owing to their controllable reversible functionality. Owing to the reversible wetting characteristics induced by change of temperature, these surfaces can be very useful for adsorption and desorption of viruses and proteins etc. in filters and chromatographic separation media. This also results in an extremely simplified process as compared to conventional separation processes. But generation of such surfaces itself can cause a high expenditure costs owing to cost of various surface preparation techniques. One alternative approach is to use the functional latex particles and subsequent grafting from these particles to achieve this goal. Preliminary trials explaining the particles adsorption and subsequent surface functionalization to achieve thermally responsive surfaces are described as follows.

Fig. (2a) shows the morphology of the particles functionalized with a thin layer of ATRP initiator (BPOEA) on the surface. As can be seen that the particles have very specific 'orange peel' morphology [14]. It was reported to be the result of colloidal instability and incompatibility of the BPOEA chains with the polystyrene particles' surface leading to random collapse of these chains on the particles' surface. Sapphire disks were used for the adsorption trials because of the ease of their surface modification to generate positive or negative charges on the surface of the disks. Any other surface with such properties would also be an equally suitable candidate for adsorption. When the particles were adsorbed on the discs, a very uneven and incomplete coverage of these discs were observed as shown in Fig. (2b). As the particles are slightly negatively charged owing to the presence of sulphate end groups from the initiator, therefore, it was suspected that these charges are responsible for repulsion forces within the particles and the carbon coated disk surface etched with plasma. Longer adsorption times of the particles on the discs also did not improve the surface coverage of the discs. In order to confirm the interactions of the particles with the disc surface, the particles were adsorbed on a disc which has been treated with positive plasma. As the particles are slightly negatively charged, therefore the positive charges on the disc surface should help to enhance the surface adsorption of the latex particles. The disc surface after particle adsorption is shown in Fig. (2c). 


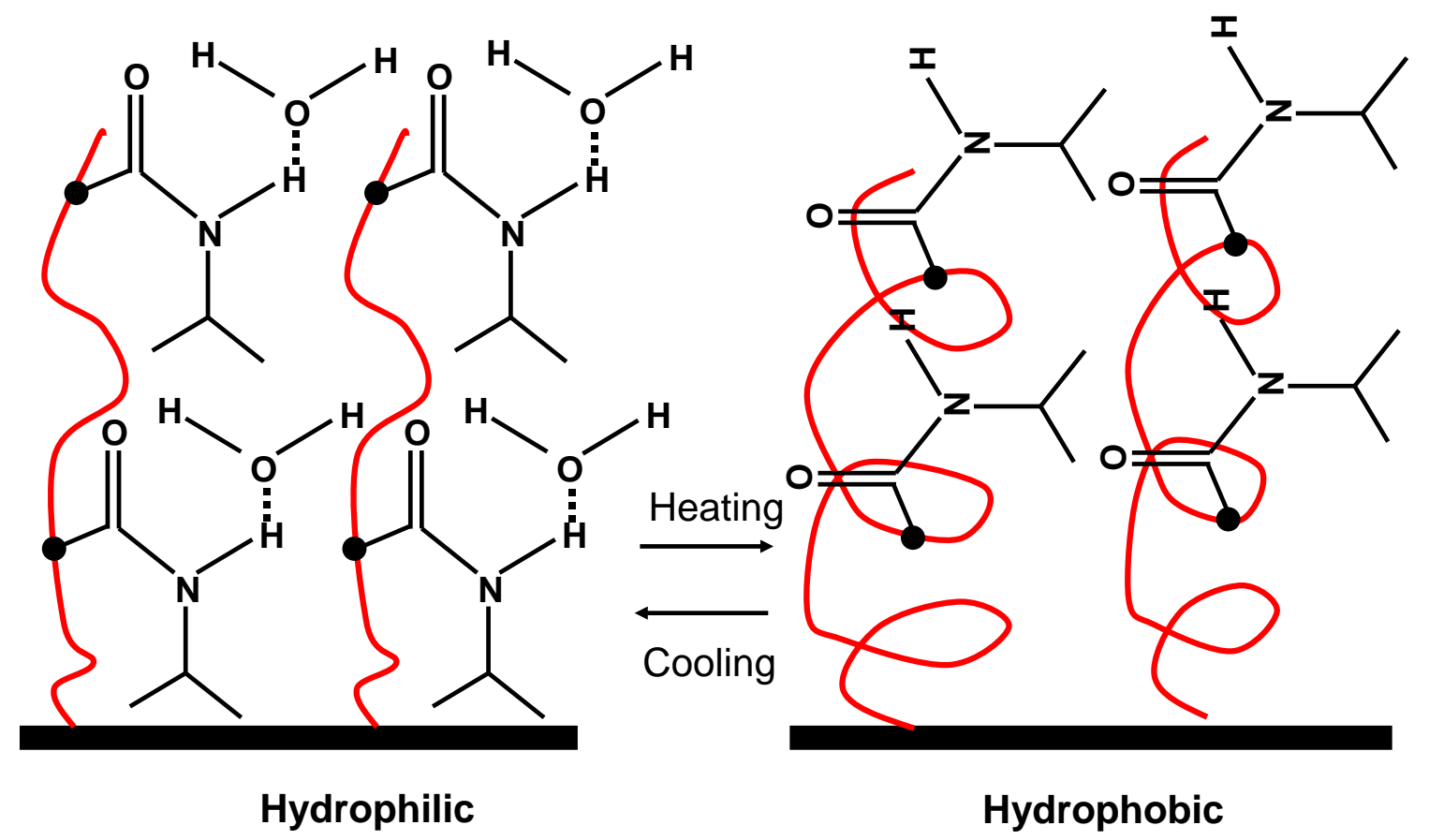

Fig. (1). Reversible hydrophilic and hydrophobic behavior of surface grafted PNIPAAM brushes below and above the LCST.

It is evident from the SEM micrograph that the adsorption of particles was greatly enhanced and the surface was almost perfectly modified. Although thin gaps could still be seen in between, but the spatial hindrance may have led to this effect. The discs were even dipped in the latex solutions for a long time to analyze the effect of loner adsorption times on the distribution. Very similar morphology was obtained as shown in Fig. (2c) indicating that the particles adsorption is very fast and the particles do not move or rearrange even if longer adsorption times are used. Thus, it confirms the successful functionalization of the disc surface with designer particles having rough and modified surfaces for further modification.

As the dead space observed in between the particle bunches is non-responsive to any further modification carried out on the surface, therefore a simple thermal treatment was provided to the discs in order to analyze the effect on surface coverage as well as subsequent properties. The disc prepared at room temperature as reported above is shown as Fig. (3a) for comparison with the discs after heat treatment. Fig. $(\mathbf{3 b}, \mathbf{c})$ show the SEM micrographs of the discs after the heat treatment of $100^{\circ} \mathrm{C}$ and $150^{\circ} \mathrm{C}$ respectively. One thing during the heat treatment should be noted that the heat treatment is meant to form a homogenous coverage of surface but the surface layer of the particles should not be affected. The disc with $100^{\circ} \mathrm{C}$ treatment indicates a better coverage as the particles have been fused with each other thus filling the gaps initially present around them. As the particles have not fused totally with each other, therefore the surface layer of ATRP initiator is expected to be more intact and not been embedded inside the melt. However, a clear flowing of melt is visible in the disc subjected to a treatment of $150^{\circ} \mathrm{C}$. The high magnification image of Fig. (3c) indicates that the particles have totally lost their structure and were totally melted and mixed with each other thus deterioration of the surface ATRP initiator layer is more likely in this case.

Atom radical transfer polymerization was used to grow the PNIPAAM brushes from the surface of the particles. Particles adsorbed on the discs at room temperature as well as at $100^{\circ} \mathrm{C}$ were used for the grafting process to analyze the effect of the heat treatment on the final surface properties. One should be careful that it is important to wash the surface thoroughly after the PNIPAAM grafting as large amount of polymer may be formed in solution and chains of this polymer can be physically adsorbed on the disk surface and can be removed during operation thus lowering the performance of the material. The behavior of water droplets placed on the PNIPAAM modified discs at room temperature and at $40^{\circ} \mathrm{C}$ is shown in the images shown in Figs. $(4,5)$. Fig. (4) indicates the behavior of the disk where the particles adsorption was achieved at room temperature. At room temperature, the droplet placed on the disc spreads quickly indicating the hydrophilic surface. On the other had, when the disc was equilibrated to $40^{\circ} \mathrm{C}$, the droplet placed on the surface did not spread out at all indicating the surface has achieved the hydrophobic character. This clearly indicates that reversible wetting characteristics on the surface could be generated. Also no change in the behavior of the droplets placed on the discs was observed for 2 minutes indicating that the behavior at particular temperature is permanent. Fig. (5) pictures the images of the water droplets on the disc which was subjected to a heat treatment at $100^{\circ} \mathrm{C}$ after particle adsorption. Close comparison indicates that a slight improvement in reversible hydrophobic and hydrophilic behavior was achieved in this case. The drop spreads out at room temperature, whereas at $40^{\circ} \mathrm{C}$, drop shrunk slightly as soon as it was placed on the disk. No change was further noticed as the drops were monitored for two minutes. The reversible behavior of the 
(a)

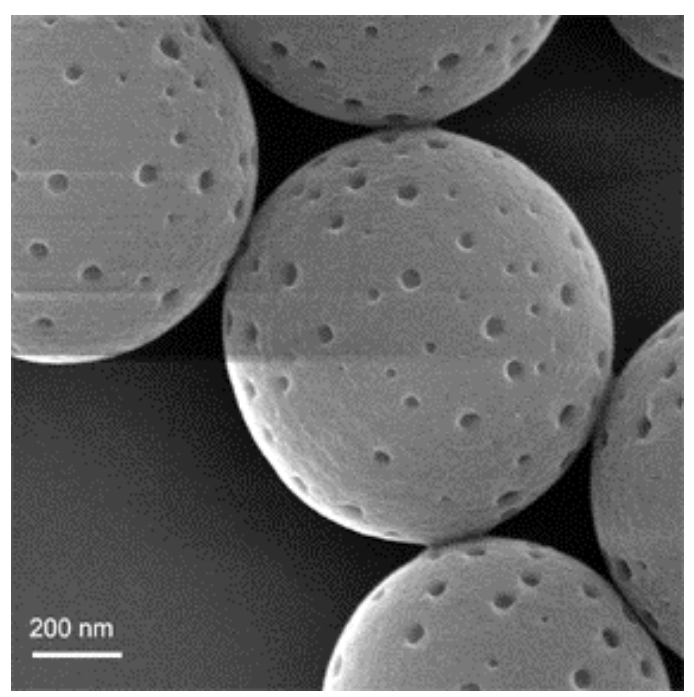

(b)

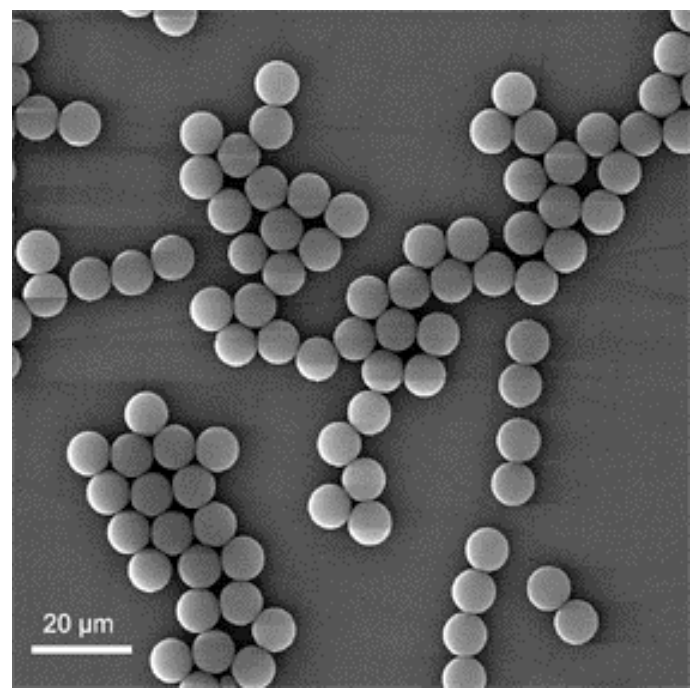

(c)

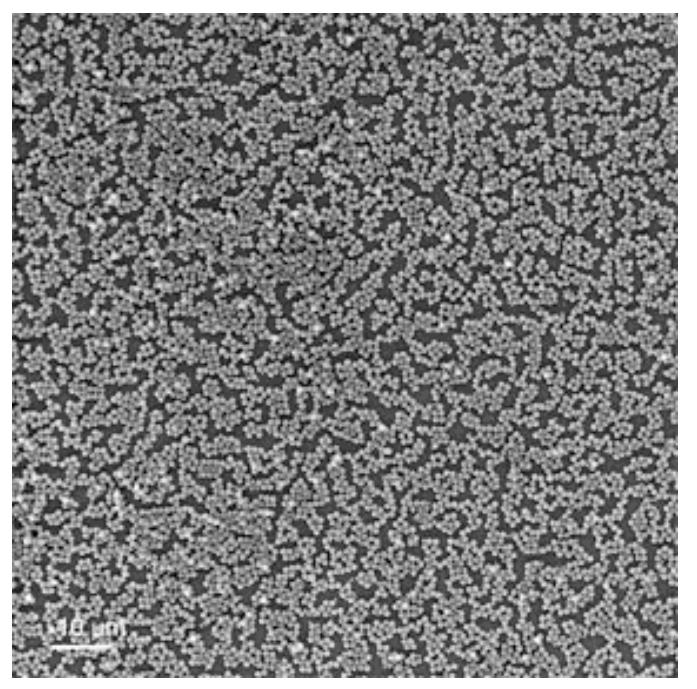

Fig. (2). (a, b) High and low magnification SEM images of the surface functionalized particles as seen on the sapphire disk and (c) SEM image representing the distribution of same particles when adsorbed on positively charged sapphire disk. (a)

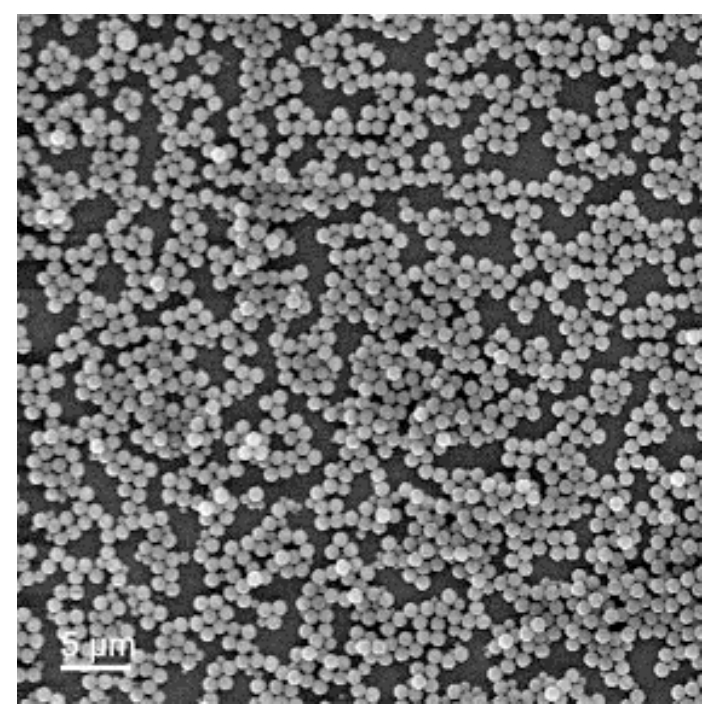

(b)

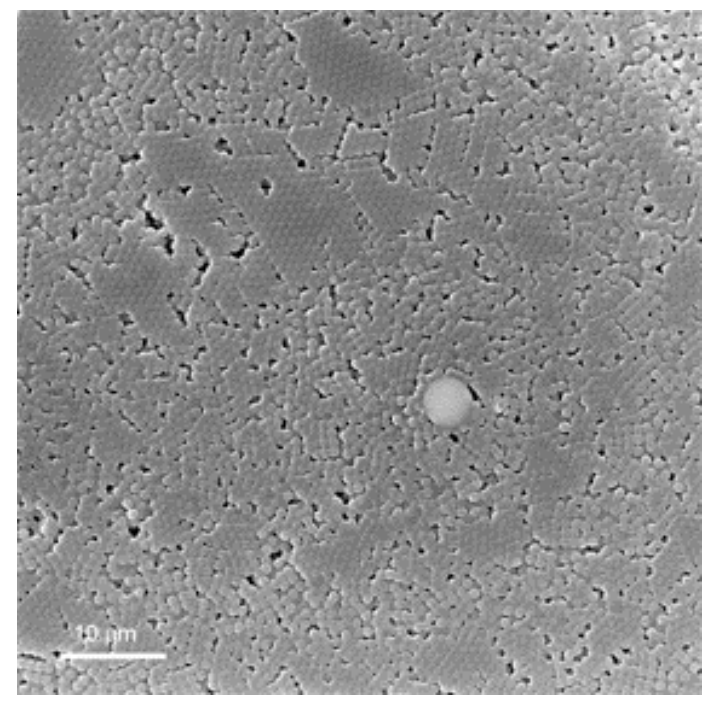

(c)

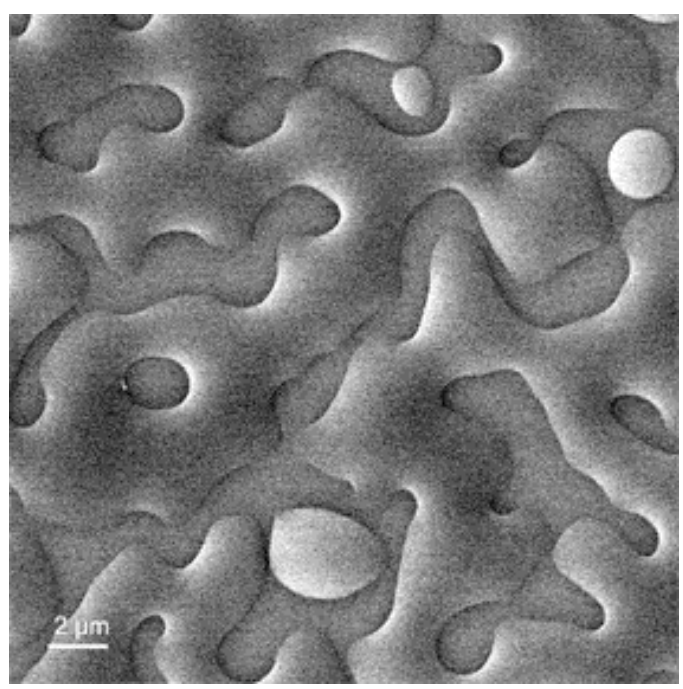

Fig. (3). SEM images showing the effect of temperature on the film of adsorbed particles when analyzed at (a) room temperature, (b) $100^{\circ} \mathrm{C}$ and (c) $150^{\circ} \mathrm{C}$. 
surfaces also indicates that the ATRP initiator layer was retained during the heat treatment. Thus reversible hydrophobic and hydrophilic surface could be achieved with relatively simpler overall process. Another important point to note here is that as the discs were washed very thoroughly after PNIPAAM grafting, therefore, the reversible properties of the surface also confirm that the particles are stably adsorbed on the surface and do not get washed away during the processing. Though the concept of generation of reversible wetting characteristics of the surface could be successfully confirmed, but the behavior of the resulting surfaces is clearly not superhydrophobic and superhydrophilic. The contact angles of the water droplets with the surfaces have also not been measured owing to similar reasons and the proof of concept has been shown only qualitatively. Further efforts are underway to achieve superhydrophobic and superhydrophilic character on the surfaces by this approach of adsorbing functionalized polymer particles, which include improvement of the surface wetting characteristics by using crosslinked particles so as to resist the heat treatment effectively thus improving the adsorption efficiency of the discs.

(a)

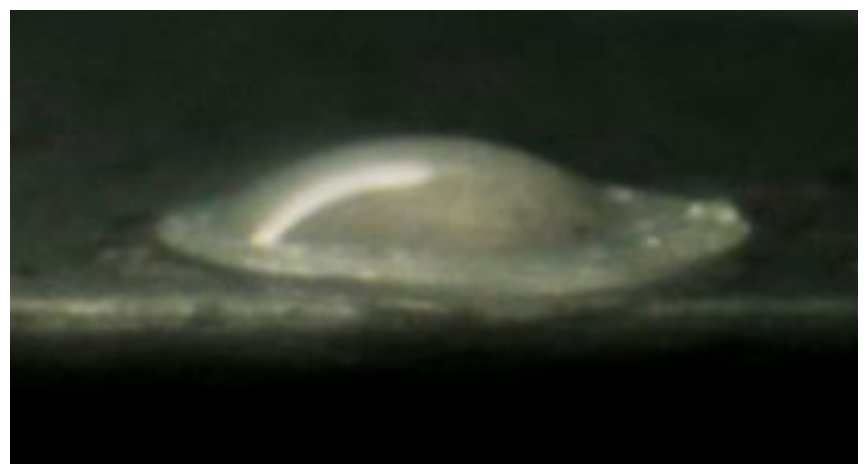

(b)

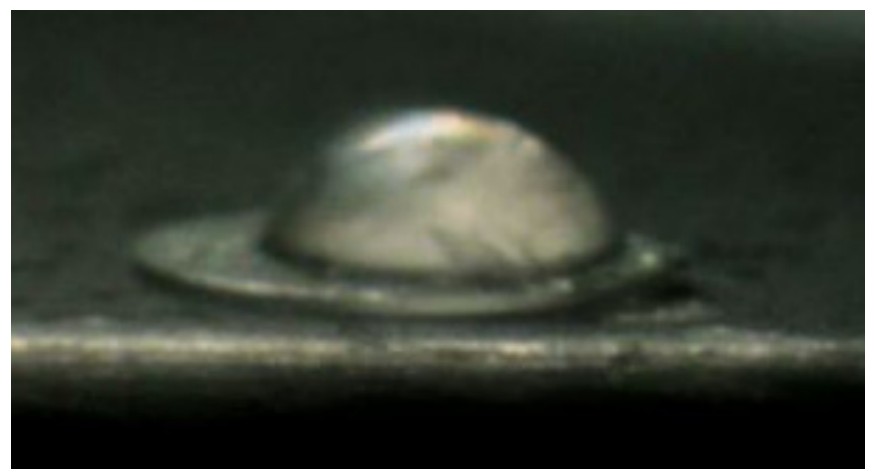

Fig. (4). Images of the water droplets on the PNIPAAM modified sapphire disk of Fig. (3a) at (a) $10^{\circ} \mathrm{C}$ and (b) $40^{\circ} \mathrm{C}$.

\section{CONCLUSIONS}

Temperature reversible surfaces could be generated successfully using a simple technique of adsorption of designer particles on the surface. The grafting of PNIPAAM brushes from the surface of these particles could subsequently be achieved. The surfaces were hydrophilic at room temperature, but turned hydrophobic as the temperature was raised to $40^{\circ} \mathrm{C}$. The heat treatment of the discs after particle adsorption improves the surface coverage with the particles and also the resulting reversible wetting behavior controlled by temperature. The surfaces are stable to rigorous washing steps and represent strong potential for use in a number of applications.

(a)

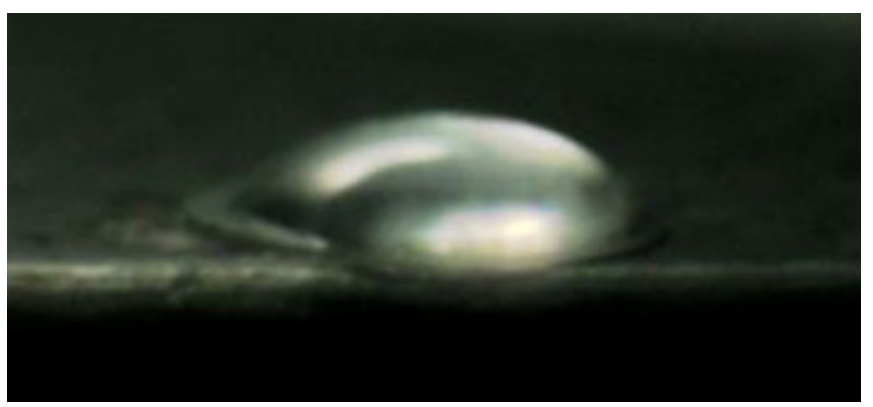

(b)

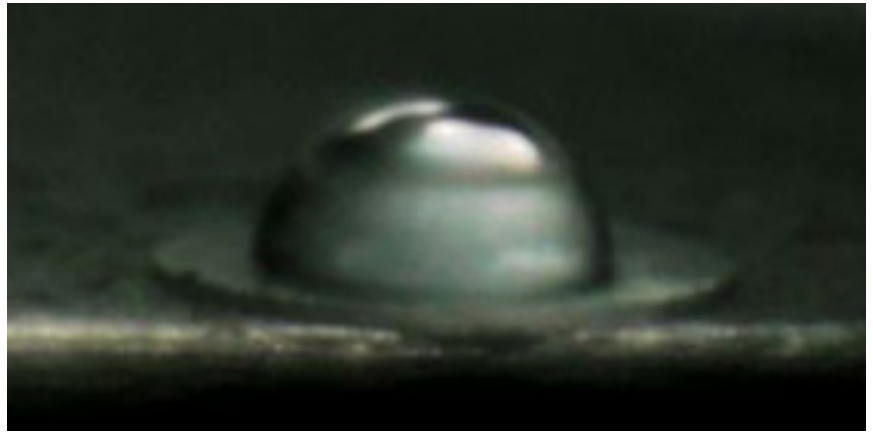

Fig. (5). Images of the water droplets on the PNIPAAM modified sapphire disk of Fig. (3b) at (a) $10^{\circ} \mathrm{C}$ and (b) $40^{\circ} \mathrm{C}$.

\section{ACKNOWLEDGEMENTS}

Expert help of Dr. Vishweshwara Herle in the study of the behavior of water droplets on the discs is greatly appreciated. The authors would like to thank Prof. M. Morbidelli and Dr. A. Butté from ETH Zurich for the scientific support for the work.

\section{REFERENCES}

[1] Ringsdorf H, Sackmann E, Simon J, Winnik FM. Interactions of liposomes and hydrophobically modified poly-(N-isopropylacrylamides): An attempt to model the cytoskeleton. Biochim Biophys Acta 1993; 1153: 335-44.

[2] Bae YH, Okano T, Kim SW. Temperature dependence of swelling of crosslinked poly(N,N'-alkyl substituted acrylamides) in water. J Polym Sci Part B Polym Phys 1990; 28: 923-36.

[3] Heskins M, Guillet JE. Solution properties of poly(N- isopropylacrylamide). J Macromol Sci Chem 1968; A2(8): 1441-5.

[4] Yoshida R, Uchida K, Kaneko Y, et al. Comb-type grafted hydrogels with rapid deswelling response to temperature changes. Nature 1995; 374: 240-2.

[5] Gao J, Wu C. The "coil-to-globule" transition of poly(N- isopropylacrylamide) on the surface of a surfactant-free polystyrene nanoparticle. Macromolecules 1997; 30: 6873- 6.

[6] Park TG, Hoffman AS. Sodium chloride-induced phase transition in nonionic poly(N-isopropylacrylamide) gel. Macromolecules 1993; 26: 5045-8.

[7] Sun T, Wang G, Feng L, et al. Reversible switching between superhydrophilicity and superhydrophobicity. Angew Chem Int Ed 2004; 43: 357-60.

[8] Extrand CW. Model for contact angles and hysteresis on rough and ultraphobic surfaces. Langmuir 2002; 18(21): 7991-9.

[9] He B, Patankar NA, Lee J. Multiple equilibrium droplet shapes and design criterion for rough hydrophobic surfaces. Langmuir 2003; 19(12): 4999-5003. 
[10] Kizhakkedathu JN, Norris-Jones R, Brooks DE. Synthesis of welldefined environmentally responsive polymer brushes by aqueous ATRP. Macromolecules 2004; 37: 734- 43.

[11] Kizhakkedathu JN, Kumar KR, Goodman D, Brooks DE. Synthesis and characterization of well-defined hydrophilic block copolymer brushes by aqueous ATRP. Polymer 2004; 45: 7471-89.

[12] Kizhakkedathu JN, Takacs-Cox A, Brooks DE. Synthesis and characterization of polymer brushes of poly $(\mathrm{N}, \mathrm{N}$ - dimethylacrylamide) from polystyrene latex by aqueous atom transfer radical polymerization. Macromolecules 2002; 35: 4247-57.

[13] Kizhakkedathu JN, Brooks DE. Synthesis of poly(N,N- dimethylacrylamide) brushes from charged polymeric surfaces by aqueous
ATRP: effect of surface initiator concentration. Macromolecules 2003; 36: 591-8

[14] Mittal V, Matsko NB, Butté A, Morbidelli M. Functionalized polystyrene latex particles as substrates for ATRP: surface and colloidal characterization. Polymer 2007; 48(10): 2806-17.

[15] Mittal V, Matsko NB, Butté A, Morbidelli M. Synthesis of temperature responsive polymer brushes from polystyrene latex particles functionalized with ATRP initiator. Eur Polym J 2007; 43(12): 4868-81.

[16] Matyjaszewski M, Gaynor SG, Kulfan A, Podwika M. Preparation of hyperbranched polyacrylates by atom transfer radical polymerization. 1. Acrylic AB monomers in "living" radical polymerizations. Macromolecules 1997; 30(17): 5192-4.

(C) Mittal and Matsko; Licensee Bentham Open.

This is an open access article licensed under the terms of the Creative Commons Attribution Non-Commercial License (http://creativecommons.org/licenses/by$\mathrm{nc} / 3.0 /$ ) which permits unrestricted, non-commercial use, distribution and reproduction in any medium, provided the work is properly cited. 